\title{
MigRACIÓN RURAL Y HUERTOS FAMILIARES EN UNA COMUNIDAD INDÍGENA DEL CENTRO DE MÉXICO
}

\author{
Margarita Cano-Ramírez ${ }^{1,5}$, Beatriz De la Tejera ${ }^{2}$, Alejandro Casas $^{1}$, Lourdes Salazar ${ }^{3}$ y \\ Raúl GARCÍA-BARriOS ${ }^{4}$ \\ ${ }^{1}$ Centro de Investigaciones en Ecosistemas, Universidad Nacional Autónoma de México \\ ${ }^{2}$ Posgrado en Desarrollo Rural, Universidad Autónoma Chapingo \\ ${ }^{3}$ Instituto Tecnológico Agropecuario de Conkal \\ ${ }^{4}$ Centro Regional de Investigaciones Multidisciplinarias, Universidad Nacional Autónoma de México \\ ${ }^{5}$ Autora para la correspondencia: jatzimay@gmail.com
}

\begin{abstract}
Resumen: Los huertos familiares evolucionan debido a la continua adecuación del espacio que ocupan, a cambios en sus componentes, así como en el entorno socio-económico y cultural de las familias que los manejan. En este estudio se tiene como hipótesis que la migración de los productores es un factor de cambio social, económico y cultural y que influirá en la estructura de los huertos, particularmente disminuyendo su riqueza y reorientándolos a una función ornamental. En una muestra de 33 huertos se registraron 287 especies perennes de plantas, principalmente con uso ornamental (58\%), medicinal (21\%) y alimentario (19\%). Mediante curvas de rarefacción y análisis estadísticos multivariados se exploraron diferencias entre huertos asociadas a la migración, pero no se identificaron disimilitudes significativas. La causa de este patrón es multifactorial, y entre los principales factores se identificaron la diversificación de estrategias de vida familiar, el carácter temporal de la migración de la gente, la resiliencia institucional y el fuerte sentido de pertenencia a la comunidad de los migrantes. Sin embargo, las actuales tendencias hacia la migración definitiva a los Estados Unidos de América y el aumento en la dependencia de los programas gubernamentales son factores que podrían alterar la relación migración - huertos familiares de acuerdo con las tendencias que se tienen como hipótesis.
\end{abstract}

Palabras clave: agroecosistemas, huertos familiares, México, migración, tlahuicas.

\begin{abstract}
Home gardens evolve due to continuous changes in their area, space, functions, and components, as well as to the dynamic circumstances of the families that manage them. People migrations induce social, economic, and cultural changes, and in this study we hypothesized that these changes influence the home gardens' composition and structure, simplifying and re-orienting them towards an ornamental function. In order to analyze this hypothesis we studied composition and structure of home gardens managed by households of the Tlahuica village of San Juan Atzingo, in the Mexican state of México. A total of 287 perennial plant species were recorded in a 33 home garden sample. The main plant uses were ornamental (58\%), medicinal (21\%), and food supply (19\%). Rarefaction curves and multivariate statistic analysis did not show significant dissimilarities between home gardens associated and not associated to migration. Causes of this pattern are multifactor, and some of the main factors identified are: family livelihood diversification, temporal migration, institutional resilience, and the migrants' strong sense of belonging to their community, which allows them to maintain communication and participation in their home town. Current trends of permanent migration to the United States of America, and increasing dependence on governmental assistance programs are risks of change in the actual relationship between the present migration patterns and the home garden structure according to the hypothesized trends.
\end{abstract}

Key words: agroecosystems, home-gardens, human migration, Mexico, Tlahuicas.

L os huertos familiares son agroecosistemas tradicionales milenarios situados alrededor de la vivienda que desempeñan un papel esencial en la vida de las familias que los manejan (Miller et al., 2006; Pulido et al., 2008). En ellos los campesinos han cultivado y domesticado una gran diversidad de especies vegetales y animales para la producción de alimentos y otros satisfactores. Los huertos generalmente están destinados a una producción moderada y temporal de una gran variedad de productos a lo largo del año (OrellanaGallego et al., 2006; Boege, 2008). También cumplen una función social y cultural al ser un lugar de encuentro, esparcimiento y bienestar para los miembros de la familia y 
sus vecinos, así como un sitio en el que se experimenta con nuevos cultivos y prácticas de manejo de plantas y animales (Kimber, 2004).

Diversos estudios en la región mesoamericana demuestran que los huertos pueden mantener altos niveles de diversidad de especies nativas y exóticas en diferentes grados de domesticación, pudiendo albergar en su interior hasta 400 especies diferentes, así como una alta diversidad intraespecífica (Casas et al., 2007; Blancas et al., 2010; Parra et al., 2010). Tan alta diversidad los convierte en importantes reservorios de recursos genéticos para diversos propósitos, que además contribuyen a mantener la resiliencia de los ecosistemas naturales (Engels, 2002; Casas et al., 2007, Pagaza-Calderón, 2009; Blancas et al., 2010; Moreno-Calles et al., 2010).

Los huertos son sistemas dinámicos que evolucionan debido a la capacidad de adaptación de las familias que los manejan a las circunstancias cambiantes del entorno socioeconómico y cultural (Peyre et al., 2006; Wiersum, 2006; Boege, 2008). Sin embargo, la capacidad adaptativa del sistema puede disminuir cuando los cambios del ambiente exceden la capacidad de las familias para sobreponerse. La comprensión de estos procesos de adaptación resulta de gran interés ante los actuales escenarios de transformación que actualmente ocurren en el ámbito rural mexicano (Moreno-Calles et al., 2010). Los efectos de esta transformación son cada vez más complejos y determinan una transición de sociedades agrarias organizadas en torno a la producción primaria, hacia sociedades rurales diversificadas. En éstas han surgido actividades como la migración y fenómenos como el abandono de prácticas productivo-culturales o el aumento de la producción agrícola con propósitos comercia-

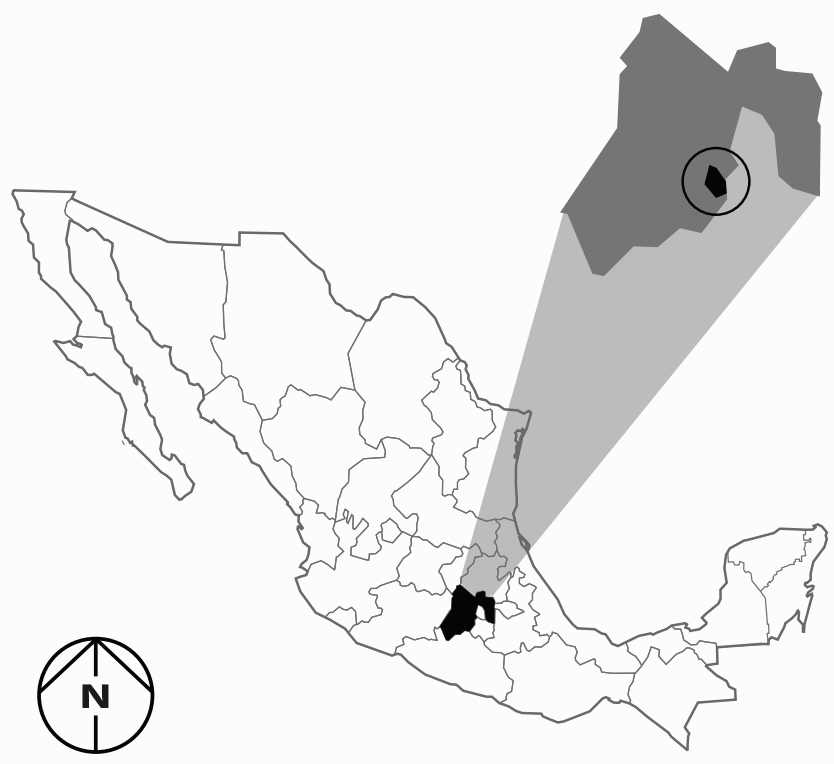

Figura 1. Mapa de localización de la comunidad de San Juan Atzingo. Fuente: Modificado de INEGI, 2010, 1900' 22.17' latitud Norte $/ 99^{\circ} 23^{\prime} 19.78^{\prime \prime}$ longitud Oeste. les, en contextos de alta incertidumbre (Pérez, 2004 en Vargas, 2009). Para algunos autores (véase por ejemplo Nair, 2006; Zárate-Vargas, 2007), tales cambios pueden poner en riesgo los sistemas agrícolas, particularmente a los huertos familiares, comprometiendo su futuro como sistemas tradicionales de conservación de la diversidad biocultural (Boege, 2008). No obstante, la diversificación de las estrategias de vida campesinas también puede contribuir a mejorar sus condiciones de vida y su capacidad adaptativa a condiciones adversas o imprevistas (Ellis y Allison, 2004). Dentro de la diversificación de actividades de las familias campesinas, la migración tiene un papel muy importante ya que en la actualidad cerca del $50 \%$ de los ingresos de los hogares rurales de los países en desarrollo proviene de esta actividad, y está ocurriendo un proceso de intensificación (De la Tejera-Hernández y García-Barrios, 2008). Arzate-Salgado y Vizcarra-Bordi (2007) reconocieron que la intensificación de este proceso migratorio en México está teniendo consecuencias en las comunidades de origen y de destino. No obstante, sus consecuencias sobre el manejo de recursos naturales en general, y de los huertos familiares en particular, apenas han comenzado a analizarse (IDRC, 2007; Guerrero-Peñuelas, 2007).

Los patrones migratorios de cada comunidad son variables en cuanto a su destino, temporalidad, número de integrantes por familia que migran, etc. por lo que cada caso es diferente. Sin embargo, los estudios que hay sobre el tema reconocen que la migración en general influye significativamente en la composición y estructura de los huertos familiares (Rebaï, 2009; Pulido et al., 2008). Ya sea por medio de la introducción de especies novedosas, conocimientos, herramientas y prácticas agrícolas en los huertos de las comunidades de destino, así como la adquisición de nuevos conocimientos derivados de la reasignación de roles familiares (Pulido et al., 2008; Aguilar-Støen et al., 2009) o por la pérdida de especies, conocimientos, herramientas y prácticas agrícolas, consecuencia de la reasignación de roles familiares, la interrupción en la cadena de transmisión de conocimientos tradicionales y procesos de aculturación (Agelet et al., 2000; Benjamin et al., 2001; Howard, 2006; Guerrero-Peñuelas, 2007; Aguilar-Støen et al., 2009). Por otra parte, Hernández-Suárez y Vásquez-García (2007) encontraron que cuando los migrantes enviaron a sus familias dinero de manera regular, la importancia del huerto en la subsistencia y bienestar disminuyó, y el huerto se reorientó a un jardín ornamental. Cuando los envíos de dinero se dieron de manera irregular la importancia del huerto aumentó sobre todo por su función en la provisión de alimentos (Guerrero-Peñuelas, 2007).

Debido a la diversidad de efectos encontrados hasta ahora. Por lo que son necesarios aún más estudios que permitan entender los patrones bajo los cuales la migración afecta el manejo de los recursos naturales, de los sistemas agrícolas y de los huertos. Particularmente, se requiere documentar el 
efecto de la intensificación que está ocurriendo en los procesos migratorios. Así, nuestro estudio se dirigió a evaluar el impacto de la intensificación del proceso migratorio de la comunidad indígena tlahuica de San Juan Atzingo sobre la riqueza, composición y estructura de los huertos. Lo anterior, con base en el supuesto de que los huertos manejados por familias con emigrantes serían menos diversos, con una estructura menos compleja y un mayor número de especies e individuos de plantas ornamentales.

\section{Materiales y métodos}

Zona de estudio. Este estudio se realizó entre 2008 y 2010 en la comunidad de San Juan Atzingo, Municipio de Ocuilan, Estado de México (Figura 1). Forma parte de una investigación más amplia denominada "Estrategias campesinas e instituciones comunitarias: hacia una propuesta agroecológica y de desarrollo local" (De la Tejera et al. datos sin publicar), la cual formó parte del macroproyecto "Manejo de ecosistemas y desarrollo humano en la Cuenca de Apatlaco-Tembembe" durante los años 2006-2008 (García-Barrios y Dávila, datos sin publicar).

La altitud a la que se encuentra su núcleo urbano oscila entre $2,513 \mathrm{~m}$ y $2,675 \mathrm{~m}$, con una superficie de 12,450 ha de bosque de pino-encino y bosque de Abies religiosa (H.B.K) Schl. en sus partes más altas. El suelo dominante es Andosol, y el clima es semifrío-húmedo, con una temperatura media anual de $15^{\circ} \mathrm{C}$ y una precipitación anual de $615 \mathrm{~mm}$ (Zarate-Vargas, 2007).

La comunidad está conformada por 2,029 habitantes repartidos en 393 unidades domésticas que están organizadas en cuatro barrios formados por linajes patrilineales (González-Ortiz, 2005; INEGI, 2010). El grado promedio de escolaridad es de 6.8 años y la población analfabeta mayor de 15 años es del 12\% (INEGI, 2010).

La tenencia de la tierra es comunal y la principal actividad económica está dada por actividades primarias que incluyen la recolección de hongos, la extracción de madera, la agricultura de autoabasto y la agricultura comercial de Daucus carota L., Opuntia ficus-indica Mill., Pisum sativum L., Rubus fructicosus L. y Vicia faba L., y, la elaboración de pulque (bebida alcohólica preparada a partir de la fermentación de savia de Agave salmiana Otto) y la cría de ganado ovino. Actividades terciarias como la carpintería, el comercio, la prestación de servicio de taxis y por la venta de fuerza de trabajo en la región y en el exterior. La migración laboral tanto nacional como internacional tiene un papel cada vez mayor dentro de las estrategias de vida diversificadas de las familias tlahuicas (De la Tejera et al. datos sin publicar).

Los habitantes de San Juan Atzingo pertenecen al grupo tlahuica, también llamado atzinca, que es uno de los grupos indígenas menos conocidos de México, pues se encuentran sólo en tres comunidades del municipio de Ocuilan en el Estado de México (González-Ortiz, 2005). El idioma hablado por los tlahuicas es el pijekak'joo que pertenece a la rama otopame de la familia otomangue (Álvarez-Fabela, 2004). En el año 2000, sólo 26 personas menores de 20 años fueron registradas como hablantes de esa lengua, por lo que se considera que ésta se encuentra en inminente peligro de extinción (INEGI, 2000; Korsbaek y Álvarez-Fabela, 2002). El 3\% de la comunidad maneja además el "tlatol", que es un término nahua para designar a la lengua ritual que se utiliza en determinadas ceremonias bajo una normativa diferente a la de las lenguas de uso cotidiano (Korsbaek y Álvarez-Fabela, 2002).

La forma de organización de la comunidad conserva raíces prehispánicas y se basa en la representación de ciudadanos de cada barrio en los cargos civiles, así como la participación rotatoria de los ciudadanos en diferentes cargos y actividades civiles, religiosas y culturales. Las instituciones locales que se han construido en la comunidad en relación con los recursos naturales y el huerto familiar son múltiples (De la Tejera, Santos y García-Barrios, datos sin publicar). Por instituciones locales entendemos al "conjunto de reglas y normas que moldean la interacción humana y las actividades repetitivas" (Appendini y Nuijten, 2008). Entre las instituciones más importantes de la comunidad se encuentran las "faenas", que son jornadas de trabajo colectivo, las mayordomías religiosas, las "brigadas forestales" cuya tarea es reforestar y prevenir incendios forestales en el bosque y la "aparecería- mediería", que se practica cuando un terreno agrícola es trabajado por su dueño y otras personas que aportan trabajo o insumos y al final se reparten los beneficios proporcionalmente entre los participantes (De la Tejera, Santos y García-Barrios, datos sin publicar). Todas estas instituciones se basan en el principio de la reciprocidad y la confianza mutua, que son principios sobre los cuales también opera el huerto familiar. Junto con la lengua, esta forma de organización conforma uno de los elementos más distintivos de la etnia tlahuica (Álvarez-Fabela, 2004).

Durante las últimas cuatro décadas el proceso migratorio se ha intensificado en la comunidad. Estimaciones de De la Tejera et al. (2008) indican que el número de migrantes pasó de cuatro personas en 1960 a 65 en 2008 (Figura 2), por lo que se calcula que en la actualidad cerca del $13.5 \%$ de la población total de la comunidad ha migrado y $47 \%$ de las unidades domésticas tienen al menos uno de sus miembros fuera de la localidad. De acuerdo con los autores referidos, $71 \%$ de los migrantes fueron hombres y tuvieron como destino los Estados Unidos, país al que ingresaron de manera ilegal para trabajar como jardineros y empleados de restaurantes. Sin embargo, este tipo de migración internacional no representa una alternativa común para la mayoría de las comunidades marginadas del país, ya que implica una alta inversión económica que no todas las familias pueden solventar. En promedio, un cruce ilegal por la frontera hasta 2008 costaba a las familias atzincas alrededor de \$3,000 dólares de Estados Unidos (Salazar-Barrientos, 2009). En el ámbi- 


\section{Migración por décadas en San Juan Atzingo}

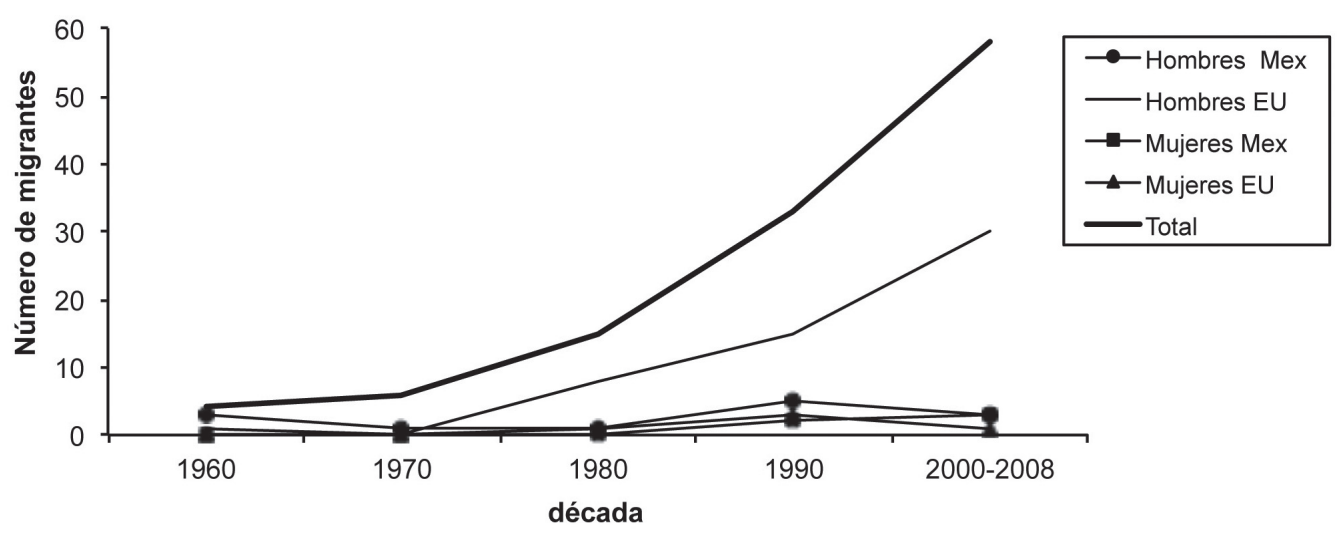

Figura 2. Migración absoluta calculada a partir de una muestra equivalente al 54\% de la comunidad. Fuente: De la Tejera-Hernández et al. (2008).

to nacional los principales destinos de los migrantes fueron las ciudades cercanas de México, Cuernavaca y Toluca, en donde los hombres se emplearon como trabajadores de la construcción y jardineros, y las mujeres como empleadas domésticas. En el $62 \%$ de los casos, la migración nacional e internacional fue temporal y los migrantes permanecieron fuera de la comunidad por menos de dos años. Sin embargo, el reforzamiento de las redes familiares y sociales, así como el endurecimiento de las políticas migratorias internacionales están favoreciendo que la migración se realice cada vez más hacia el extranjero de manera permanente (Salazar-Barrientos, 2009).

Colecta de datos. El trabajo de campo se realizó de 2008 a 2010. Se seleccionó una muestra de 33 unidades domésticas (UD) con huerto familiar que representaron el $10 \%$ del total de huertos de la comunidad de acuerdo a los datos disponibles en ese momento. La muestra fue dirigida y se seleccionaron aquellas UD participantes en los proyectos "Estrategias campesinas e instituciones comunitarias: hacia una propuesta agroecológica y de desarrollo local" y/o "Reconversión agroecológica e industrialización artesanal de chícharo y nopal" (De la Tejera et al. datos sin publicar), porque se contaba con mayor información socio-económica y una relación de confianza con tales UD.

Se consideró el estatus migratorio de la UD, dividiendo la muestra en aquellos huertos manejados por familias con al menos un integrante migrante y aquellos manejados por familias sin migrantes. Se incluyeron en la muestra huertos de los cuatro diferentes barrios de la comunidad, pues éstos representaron distintas condiciones ambientales y culturales que se consideró podían influir en el manejo del huerto (Cuadro 1). Para diferenciar las UD migrantes de las no migrantes se siguió el criterio utilizado por Salazar-Barrientos (2009), quien consideró como UD migrantes a aquellas con al menos un miembro que hubiera salido de la comunidad por motivos laborales entre 2005 y 2008. En cada huerto visitado se registró el número total de especies de plantas útiles y el número de individuos por especie, su talla y ubicación dentro del huerto. Los análisis de composición y estructura del huerto se basaron en el registro de las especies perennes. Se realizaron recolectas de ejemplares que posteriormente se prepararon como especímenes de herbario para su identificación botánica; los ejemplares colectados fueron identificados por personal del Jardín Botánico del

Cuadro 1. Características de los huertos de acuerdo a su ubicación dentro de los barrios de la comunidad. En donde mixto corresponde a presencia de indígenas y mestizos con predominancia de indígenas, y el entrecomillado a la forma de nombrarse a si mismos y por el resto de la comunidad.

\begin{tabular}{|c|c|c|c|c|}
\hline Barrios & Loma de Teocaltzingo & El Atorón & Centro & Nativitas \\
\hline Altitud (m) & 2,675 & 2,674 & 2,560 & 2,513 \\
\hline Presencia de heladas & si & si & no & no \\
\hline Pendiente de los huertos & regular & pronunciada & baja & baja \\
\hline Colindancia con el bosque & alta & alta & media & baja \\
\hline Autoadscripción étnica & $\begin{array}{l}\text { "los verdaderos tlahuicas", } \\
\text { "los más indios" }\end{array}$ & tlahuicas & tlahuicas & tlahuicas \\
\hline Número de unidades domésticas & 120 & 23 & 159 & 91 \\
\hline
\end{tabular}


CIEco-UNAM. Los muestreos se hicieron en compañía de la propietaria, o en su ausencia por algún otro miembro de la Unidad Doméstica, para recuperar los nombres locales de las especies así como sus usos y lugares de procedencia.

Análisis y procesamiento de datos. La diferencia en riqueza de especies se evaluó comparando los promedios esperados de especies perennes en huertos con migrantes y sin migrantes, calculado con base en curvas de rarefacción elaboradas para cada uno de los huertos en el menor número de individuos observado. Se usó el programa Estimates versión 8.0 (Gotelli y Colwell, 2001; Magurran, 2004).

La similitud entre huertos se examinó a partir de una matriz de datos de abundancia por especie por huerto y se efectuaron análisis de conglomerados en el programa Biodiversity Pro (versión 2.0). La equitatividad entre huertos se comparó a partir de las curvas de rango de abundancia generadas a partir del ordenamiento decreciente de la abundancia relativa de las especies de los huertos con migrantes y los huertos sin migrantes (Magurran, 2004).

Para establecer si había diferencias en el uso de las plantas asociadas a la migración se utilizó la prueba de KruskalWallis para datos no paramétricos. Esta misma prueba fue utilizada para evaluar si existían diferencias entre huertos con migrantes y sin migrantes en la estructura vertical de los huertos, el origen de las plantas y las formas de manejo de éstas. Las formas de manejo de las plantas se establecieron de acuerdo con lo propuesto por Casas et al. (1996). Las plantas cultivadas son aquellas que el propietario siembra, propaga vegetativamente o transplanta; las protegidas son aquellas que se establecen naturalmente en el huerto y que el propietario decide proteger y propiciar; finalmente, las plantas toleradas son aquellas que se establecen de manera natural en el huerto y que el dueño no propicia ni protege, pero tampoco remueve.

\section{Resultados}

Riqueza y estructura de los huertos familiares. Los huertos de San Juan Atzingo son sistemas heterogéneos y diversos. En conjunto, la muestra albergó un total de 287 especies perennes y 85 familias (Apéndice). La familia Asteraceae fue la más abundante con 32 especies, seguida de Rosaceae (18), Crassulaceae (15), Cactaceae (14) y Lamiaceae (11). A continuación se describen las características del huerto así como el arreglo espacial de las especies vegetales.

El huerto típico de San Juan Atzingo o "lote", como lo llaman sus pobladores, tiene un tamaño promedio de $964 \pm$ $882 \mathrm{~m}^{2}$ en huertos sin migrantes y $819 \pm 663 \mathrm{~m}^{2}$ en huertos con migrantes. Tradicionalmente se compone de un patio central alrededor del cual se establece la vivienda parental y las viviendas de los hijos varones y sus nuevas familias. En el huerto también se ubican el fogón de leña y la letrina. Por lo regular el patio tiene piso de tierra y una superficie libre que sirve como espacio de reunión y preparación de alimen- tos para las festividades y celebraciones comunitarias. En las orillas y el frente de la vivienda, el patio tiene las plantas "más vistosas", frecuentemente especies ornamentales en macetas o jardineras. En la parte posterior de las casas se encuentran el resto de las plantas del huerto, la mayoría de ellas sembradas directamente en el suelo y destinadas a las distintas necesidades de la familia como alimento y medicina, entre otras. Las plantas que requieren de mayores cuidados, como las hierbas empleadas como condimentos o medicinales, están próximas a la casa habitación y cuentan con cercados de diversos materiales para evitar que las aves de corral y el ganado ovino las dañen. Los árboles se localizan en las partes más lejana a la casa, y junto con los magueyes pulqueros (Agave salmiana) y la zarza silvestre (Rubus fructicosus), pueden servir como cerco vivo o lindero. La mayoría de los huertos colindan con otra unidad productiva o de prestación de servicios de la misma familia, como milpas, nopaleras, huertas de flores o frutas, carpinterías y más recientemente invernaderos de plantas de ornato. Este patrón muestra las estrategias de diversificación productiva, pero es importante precisar que se encontraron casos en los que los invernaderos sustituyeron parcial o totalmente la superficie del huerto.

De la década de los 90 a la fecha, las viviendas tradicionales de madera con dos habitaciones y cocina de leña han comenzado a sustituirse por viviendas con paredes de ladrillo y cemento que incluyen la letrina y la cocina con gas. Sin embargo, el fogón de leña es un elemento que coexiste con la estufa de gas L.P., ya sea por el gusto de los propietarios por cocinar con leña o por el elevado precio del gas.

La estructura horizontal de las especies dentro de los huertos atzincas es variable. Sin embargo, se identificó un patrón general que coincide con lo registrado en otros huertos mesoamericanos en las que existe un área para las plantas de ornato, un área para las plantas medicinales y hierbas aromáticas, otra área para los frutales y cultivos anuales y finalmente un área para los animales domésticos (véase Ruenes-Morales, 1993; Pulido et al., 2008).

En cuanto a su estructura vertical se encontraron cuatro estratos: el estrato herbáceo bajo con plantas menores a 30 cm que incluyó $31 \%$ de los individuos vegetales de los huertos. En su mayoría fueron de uso medicinal y ornamental, con especies como Melissa officinalis L., Origanum vulgare L., Viola tricolor L. y algunas cactáceas y crasuláceas. El de las plantas herbáceas intermedias, de $31 \mathrm{~cm}$ a $1.5 \mathrm{~m}$ que comprendió 53\% de los individuos y estuvo conformado por especies como Lilum candidum var. peregrinum Baker, Ruta graveolens L. Salvia gesneriflora Lindl y Zantedechia aethiopica $\mathrm{L}$. El de las plantas herbáceas y arbustivas de $1.5 \mathrm{~m}$ a $5 \mathrm{~m}$ de alto, que incluyó $14 \%$ de los individuos, con plantas alimentarias como Rubus fructicosus, Prunus persica (L.) Batsch y plantas ornamentales como Datura sanguinea L. y finalmente, el estrato de árboles mayores de $5 \mathrm{~m}$ de altura que sólo abarcó al 1.6\% de los individuos con 
especies como Abies religiosa, Cupressus lindleyi Klotzsch ex Endl., Persea americana L, y varias especies de Pinus. No se encontraron diferencias significativas en la estructura horizontal y vertical entre huertos con migrantes y sin migrantes $(P>0.05)$.

Composición florística y estatus migratorio de la UD. El número total de especies perennes en los huertos sin migrantes fue de 238 mientras que en los huertos con migrantes fue de 217. El número de especies por huerto fue muy variable; en promedio, los huertos sin migrantes tuvieron $38.05 \pm 20.17$ especies y los huertos con migrantes 42.25 \pm 11.9 especies. La riqueza esperada para 16 individuos calculadas a partir de las curvas de rarefacción en los huertos sin migrantes fue de $14.2 \pm 1.1$ mientras que para los huertos con migrantes fue de $15.9 \pm 1.2$. No se encontraron diferencias significativas entre los huertos con migrantes y sin migrantes $(P>0.05)$.

La mayoría de las especies tuvo una frecuencia baja. En los huertos sin migrantes sólo tres de las 238 especies perennes se encontraron en más del $75 \%$ de los huertos, mientras que el $45 \%$ de las especies estuvo presente en un sólo huerto. En el caso de los no migrantes las proporciones fueron parecidas ya que únicamente cuatro de las 217 especies estuvieron en más del $75 \%$ de los huertos y el $44 \%$ de las especies tuvieron presencia en un sólo huerto. Las curvas de rango-abundancia (Figura 3) indican que, independientemente del estatus migratorio, los huertos están dominados por unas pocas especies con muchos individuos y una gran mayoría de especies con pocos o incluso una única planta en los huertos. En general, las especies más abundantes fueron plantas ornamentales exóticas. En los huertos sin migrantes Agapanthus sp., fue la especie más dominante $(\mathrm{Pi}=0.178)$, seguida de Zantedechia aethiopica $(\mathrm{Pi}=0.108)$, Euonymus japonicus Thunb. $(\mathrm{Pi}=0.061)$, Pelargonium $x$ domesticum Baile $(\mathrm{Pi}=0.032)$ y Rosa sp. $(\mathrm{Pi}=0.030)$. En los huertos

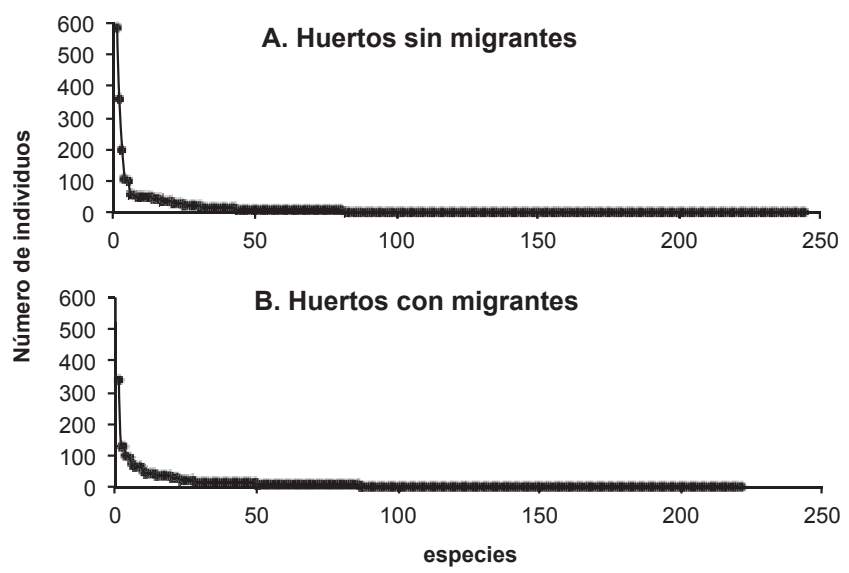

Figura 3. Curvas rango-abundancia para huertos con migrantes y sin migrantes; A) el pico de mayor abundancia (> 100 individuos) está dado por cinco especies; B) el pico de mayor abundancia (> 100 individuos) está dado por cuatro especies.

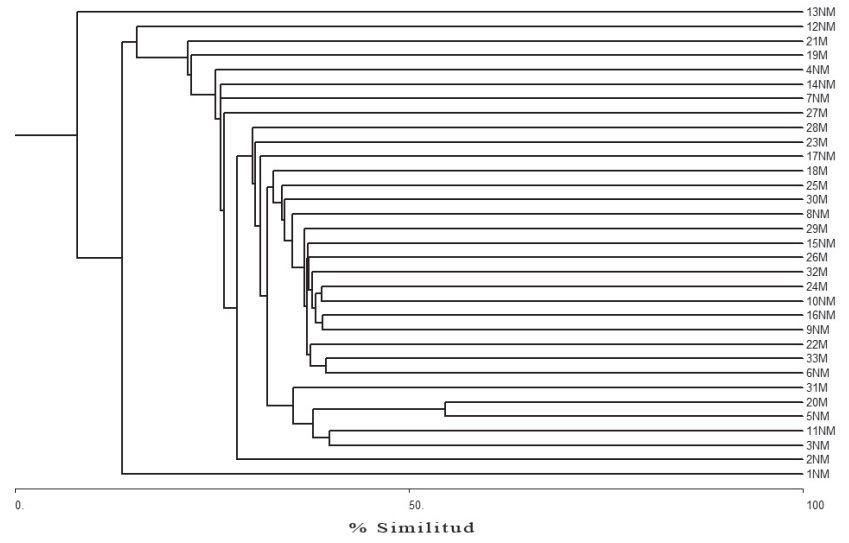

Figura 4. Análisis de conglomerados de Bray-Curtis de los huertos familiares de SJA. En donde $\mathrm{NM}=$ huertos sin migrantes y $\mathrm{M}=$ huertos con migrantes.

con migrantes las plantas dominantes fueron Agapanthus sp. $(\mathrm{Pi}=0.129)$, Pelargonium $x$ domesticum $(\mathrm{Pi}=0.049)$, Lilum candidum var. peregrinum $(\mathrm{Pi}=0.048)$ y Rosa sp. $(\mathrm{Pi}$ $=0.037$ ).

El análisis de conglomerados mostró que los huertos atzincas tienen composiciones de especies particulares y son sistemas muy heterogéneos y diferentes entre sí. No se encontró ningún patrón de agrupamiento asociado a la migración (Figura 4). Sin embargo, los huertos sin migrantes $13 \mathrm{NM}, 1 \mathrm{NM}$ y $12 \mathrm{NM}$ se diferenciaron del resto a un nivel muy bajo de similitud, debido a su escasa diversidad y presencia de especies raras, en su mayoría ornamentales. Por ejemplo el huerto 13NM presentó especies exclusivas como Aquilegia vulgaris L., Hypericum calycinum L. y Pyrus malus L., así como especies raras que compartió únicamente con otro huerto (Cassia sp., Cestrum sp., Hamelia sp., Heimia salicifolia Link \& Otto, Kalanchoe tomentosa Baker, Piqueria trinervia Cav. y Stevia salicifolia Cav. var salicifolia). El huerto 1NM tuvo tres especies raras compartidas únicamente con otro huerto (Beaucarnea recurvata Lam., Chamaecereus silvestrii Britton \& Rose y Cuphea hyssopifolia Kunth). El huerto 1NM fue el segundo menos diverso. El huerto 12NM tuvo como especie exclusiva Ananas comosus L. siendo el huerto menos diverso.

Las UD de estos tres huertos fueron atípicas; pues tendieron a la especialización y sus ingresos principales dependieron de actividades que implican una baja dependencia de los recursos naturales nativos locales y del huerto, lo que se reflejó en el poco interés en mantener un huerto diverso y multifuncional.

Propósito del huerto. Sin importar el estatus migratorio de la familia, las principales razones expresadas por las mujeres para tener plantas en sus huertos se concentraron en beneficios intangibles como que las plantas son indispensables para que una casa se vea "bonita", "adornada" y que "donde no hay plantas no hay mujeres", por lo que el 58\% 
Cuadro 2. Promedio (mínimo-máximo) de plantas que hay en los huertos por categoría de uso y categoría migratoria de sus integrantes. Acrónimos: $\mathrm{HF}$ tot. $=$ total de huertos muestreados, $\mathrm{HF}$ sin mig. $=$ huertos sin migrantes, $\mathrm{HF}$ con mig $=$ huertos con migrantes. n.s. $=(P>0.05)$.

\begin{tabular}{|c|c|c|c|c|c|c|c|c|}
\hline & Medicinal & Alimentario & Ornato & Ritual & Sombra & Cerco vivo & Leña & Forraje \\
\hline HF tot. & $\begin{array}{l}5.6 \\
(0-17)\end{array}$ & $\begin{array}{l}11.7 \\
(0-20)\end{array}$ & $\begin{array}{l}23.6 \\
(6-50)\end{array}$ & $\begin{array}{l}0.6 \\
(0-3)\end{array}$ & $\begin{array}{l}0.9 \\
(0-4)\end{array}$ & $\begin{array}{l}0.3 \\
(0-2)\end{array}$ & $\begin{array}{l}0.3 \\
(0-1)\end{array}$ & $\begin{array}{l}0.1 \\
(0-1)\end{array}$ \\
\hline HF Sin mig & $\begin{array}{l}5.6 \\
(0-17)\end{array}$ & $\begin{array}{l}11.2 \\
(0-18)\end{array}$ & $\begin{array}{l}23.1 \\
(6-50)\end{array}$ & $\begin{array}{l}0.7 \\
(0-3)\end{array}$ & $\begin{array}{l}0.9 \\
(0-4)\end{array}$ & $\begin{array}{l}0.2 \\
(0-2)\end{array}$ & $\begin{array}{l}0.3 \\
(0-1)\end{array}$ & $\begin{array}{l}0.0 \\
(0-0)\end{array}$ \\
\hline HF Con mig & $\begin{array}{l}5.6 \\
(1-13)\end{array}$ & $\begin{array}{l}12.3 \\
(8-20)\end{array}$ & $\begin{array}{l}24.3 \\
(12-46)\end{array}$ & $\begin{array}{l}0.5 \\
(0-2)\end{array}$ & $\begin{array}{l}1.1 \\
(0-3)\end{array}$ & $\begin{array}{l}0.3 \\
(0-2)\end{array}$ & $\begin{array}{l}0.3 \\
(0-1)\end{array}$ & $\begin{array}{l}0.1 \\
(0-1)\end{array}$ \\
\hline$\chi^{2}$ & $0.19^{\text {n.s. }}$ & $0.047^{\text {n.s. }}$ & 0.469 n.s. & $0.58^{\text {n.s. }}$ & $0.251^{\text {n.s. }}$ & $0.231^{\text {n.s. }}$ & $0.013^{\text {n.s. }}$ & $1.06^{\text {n.s. }}$ \\
\hline
\end{tabular}

de las plantas tuvieron un uso ornamental. Las plantas también se consideraron importantes para alejar las "envidias" y hacerlas "olvidar sus penas" además de ser un reflejo del cuidado que pone su propietaria a su casa, por lo que entre más plantas se tienen, se interpreta que la mujer cumple mejor con su rol de cuidar el hogar. El tener una gran cantidad de plantas diferentes también fue un motivo de orgullo para las mujeres atzincas.

En segundo término quedaron razones asociadas a beneficios tangibles como "las plantas del huerto son necesarias e indispensables como alimento y remedio para la familia", por lo que las plantas medicinales $(21 \%)$ y las alimentarias (19\%) representaron una proporción más reducida que la de las plantas ornamentales. Las plantas rituales, para sombra, cerco vivo, leña, utensilios y forraje representaron el $5 \%$ del total de especies. Estas razones nos indican que en el diseño de los huertos atzincas prevalecen los valores intangibles respecto a los pragmáticos, en donde la diversidad aparece como un valor muy destacado en sí mismo. Cuando hablamos de diversidad nos referimos la diversidad de especies vegetales del huerto, pero también a las múltiples combinaciones que hay entre ellas, a la diversidad de estrategias productivas y de manejo de los recursos vegetales, y a los numerosos arreglos espaciales si bien como ya se describió anteriormente, hay ciertas generalidades en estos arreglos. No se encontraron diferencias significativas en el uso de las plantas asociadas sólo a la migración (Cuadro 2).

Manejo del huerto. El 75\% de las especies de plantas fueron cultivadas, el $13 \%$ protegidas y el $12 \%$ toleradas. No hubo diferencias asociadas al estatus migratorio del huerto. Los resultados reflejan la intencionalidad por mantener las plantas en los huertos, pero es interesante que muchas de ellas no tuvieron una finalidad utilitaria, como en el caso de las toleradas, sino que se les conservó por que hay una visión del uso potencial de sus plantas: aunque no se les usa ahora, se les puede llegar a necesitar. Este patrón es compatible con procesos migratorios estacionales y no lo es con la migración permanente de todos los integrantes de la familia.

El $56 \%$ de las especies fueron exóticas, el $27 \%$ nativas de los ecosistemas locales y el $17 \%$ nativas para la región de
México y Centroamérica.

La forma en que las familias obtuvieron las plantas que componen sus huertos también fue muy diversa. Los ejemplares se adquirieron por medio de la compra en los mercados regionales y los invernaderos de la comunidad, el intercambio y regalo entre vecinos y familiares dentro de la misma comunidad o la recolecta y transplante de ejemplares silvestres procedentes de los alrededores. También se encontraron ejemplares provenientes de otros estados del centro y occidente así como del norte del país. Frecuentemente estas plantas fueron un obsequio o intercambio con personas de la propia unidad doméstica o personas allegadas a ella, que emigró a trabajar a esos sitios.

\section{Discusión}

Los huertos de San Juan Atzingo son sistemas activos y dinámicos que cuentan con una gran diversidad de especies (Cuadro 3), numerosos usos de las plantas y una compleja estructura horizontal y vertical.

Su estudio nos permitió observar una identidad renovada en el sentido de que la diversidad que sostienen ya no sólo proviene de un origen étnico único, sino de los constantes procesos de transformación que ha sufrido la comunidad, de los constantes flujos de su población y los permanentes intercambios que sostienen con sus entornos cercanos y lejanos a través de los procesos migratorios. En los que se han intercambiado especies vegetales, conocimientos e ideas, dando lugar a expresiones multiculturales que se reflejan en la actual estructura y composición de los huertos.

Consideramos que la diversificación de actividades, aplicada en todas las esferas de la vida cotidiana de las unidades domésticas de esta comunidad, es uno de los factores que ha contribuido a amortiguar los efectos de la migración sobre la composición y estructura de los huertos familiares.

La diversificación de actividades ha sido posible entre los tlahuicas debido a que las familias tienen un promedio de 6.7 integrantes, y una base de recursos, naturales y económicos, que son aprovechados por los miembros de la UD de muy distintas maneras y sin que la ausencia de algunos 
Cuadro 3. Comparación de la riqueza de especies vegetales provenientes de los huertos familiares de diferentes grupos indígenas mexicanos y su aportación a la conservación de la flora local. En donde d.n.d corresponde a datos no disponibles y las plantas nativas pueden hacer referencia a especies nativas para Mesoamérica o para los agroecosistemas locales en donde se ubican los huertos, sin embargo no en todos los casos se especifica el criterio utilizado.

\begin{tabular}{|c|c|c|c|c|c|c|}
\hline Autor & Clima & grupo étnico & $\begin{array}{l}\text { Huertos } \\
\text { estudiados }\end{array}$ & $\begin{array}{l}\text { Promedio de } \\
\text { spp. por huerto }\end{array}$ & $\begin{array}{l}\text { Total de spp. } \\
\text { registradas }\end{array}$ & $\%$ nativas \\
\hline Cano-Ramírez et al. (este artículo) & Templado & tlahuica & 33 & 40.7 & 287 & 27 \\
\hline Del Ángel-Pérez y Mendoza-B, 2004 & Templado sub-húmedo & totonaco & 40 & d.n.d & 223 & d.n.d \\
\hline Chi-Quej, 2009 & Calido-subhúmedo & chol & 26 & d.n.d & 201 & d.n.d \\
\hline De Clerck y Negreros-Castillo, 2000 & Cálido-húmedo & maya & 78 & d.n.d & 80 & d.n.d \\
\hline García De Miguel, 2000 & Cálido húmedo & maya & 300 & 21.5 & 156 & 74 \\
\hline Caballero, 1992 & Cálido-húmedo & maya & 60 & d.n.d & 83 & 32 \\
\hline Vogl et al., 2002 & Templado & tzotzil & 30 & $25-82$ & 241 & 20.7 \\
\hline Basurto-Peña, 1982 & Cálido-húmedo & nahua & 6 & 65.5 & 295 & d.n.d \\
\hline Manzanares-Medina et al., 2009 & Semi-cálido & zapoteco & 10 & 31.2 & d.n.d & d.n.d \\
\hline Allison, 1983 & d.n.d & nahua & 4 & 33 & 82 & 51 \\
\hline Cano-Ramírez, 2003 & Cálido sub-húmedo & mixteco & 10 & 30.3 & 129 & 56 \\
\hline
\end{tabular}

de sus miembros dificulte el funcionamiento de la UD. Así los distintos miembros de la unidad doméstica pueden integrarse como trabajadores en la economía local, mientras que otros lo pueden hacer en diferentes lugares del país o incluso fuera de él, y enviar dinero a la unidad doméstica (remesas). Estas remesas significan una aportación importante al ingreso de las UD de San Juan Atzingo, 24\% del ingreso está dado por remesas de EU y $13 \%$ por remesas nacionales, pero no son la única fuente de ingresos, ni la más importante, como ocurre en otras comunidades en donde éstas pueden llegar a representar hasta el $80 \%$ del ingreso total de las UD (De la Tejera et al. datos sin publicar). El que el ingreso no esté focalizado en la migración ha repercutido en que la actividad agrícola, en donde se incluyen los huer-

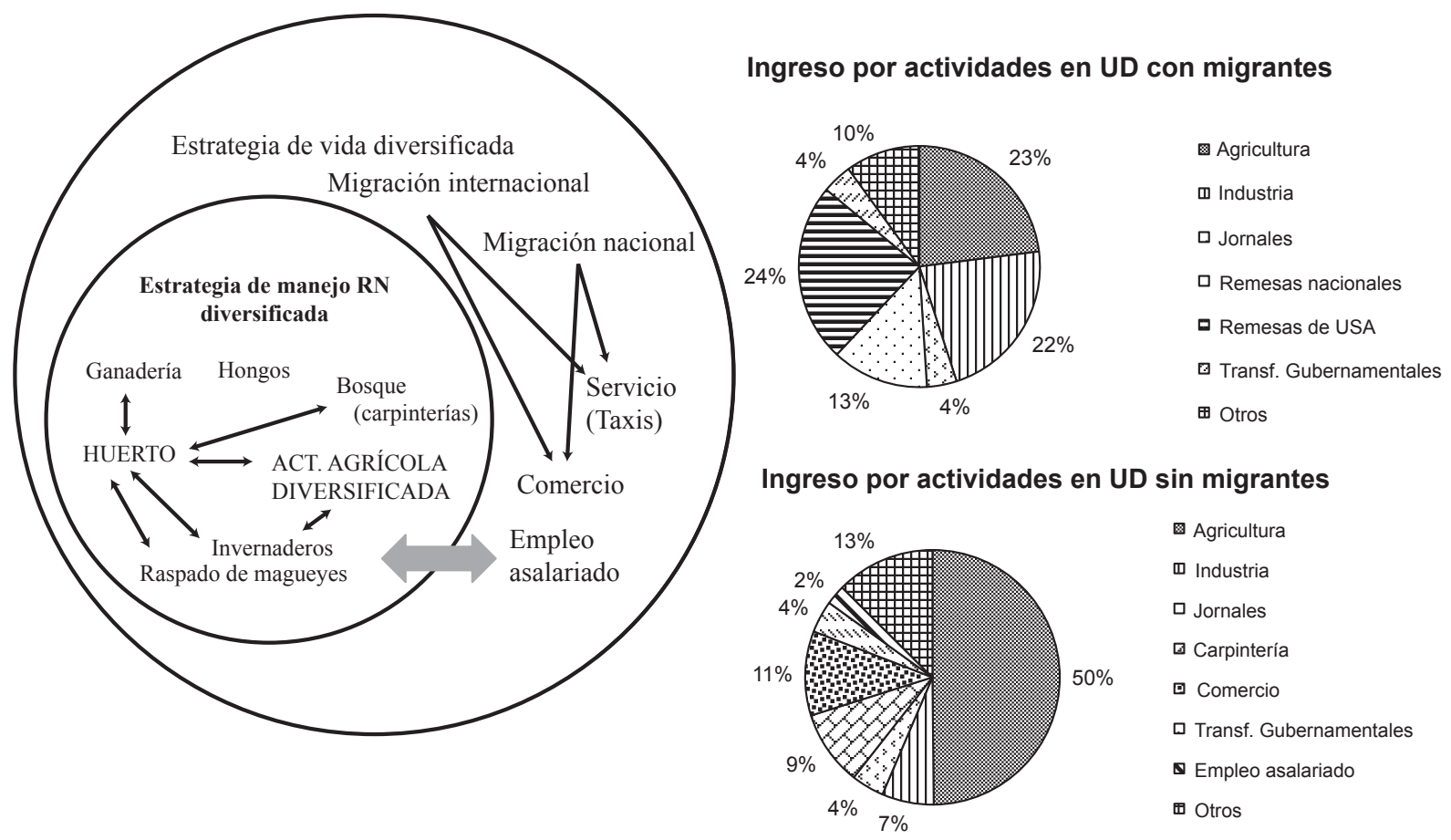

Figura 5. Diagrama de las estrategias de vida de las familias de San Juan Atzingo. Fuente: modificado de De la Tejera-Hernández et al. (2008). 
tos familiares, no haya disminuido como en otros sitios del país. La diversificación de actividades también provocó que no se observaran diferencias en la utilización de las plantas del huerto, ya que no hubo necesidad de compensar la incertidumbre de las remesas por medio de la introducción de plantas alimentarias como ocurrió en el caso de los huertos mazahuas de San Felipe del Progreso en el Estado de México (Guerrero-Peñuelas, 2007).

La temporalidad de la migración es otro factor que consideramos determinante en la ausencia de diferencias en la composición y estructura de huertos con migrantes y $\sin$ migrantes. El $62.3 \%$ de los migrantes fueron migrantes temporales, en su mayoría hombres jóvenes, que se fueron con la idea de juntar dinero para poder iniciar una familia o para mejorar las condiciones de vida de la familia que estaban comenzando. Pero siempre migraron con la idea de regresar y establecerse de manera permanente en la comunidad (Salazar-Barrientos, 2009). Esta motivación les permitió mantener el apego y los vínculos con la comunidad, así como el flujo de conocimientos sobre el huerto y sus prácticas de generación en generación. La elección de ciertas especies vegetales por ejemplo, está asociada a su uso en las celebraciones de la comunidad, a los alimentos que se consumen en la vida diaria y a las especies que de generación en generación se han utilizado para tratar las enfermedades de la familia y que la migración temporal permite reproducir. Los vínculos que los migrantes tienen con su comunidad de origen se ven reflejados en su participación en los distintos cargos religiosos y civiles, que es un factor indispensable para la reproducción de la cultura atzinca, ya que justamente son las fiestas y la forma de organización de la vida comunitaria lo que los distingue del resto de los pueblos vecinos y les ha ayudado a formar una identidad propia (ÁlvarezFabela, 2004), si bien hay que entender esta identidad, como lo hemos indicado, es un producto dinámico y no estático, de los procesos multiculturales, de flujos poblacionales y procesos educativos complejos que ha vivido la población de San Juan Atzingo en las décadas recientes.

Finalmente las fuertes redes sociales que existen al interior de la comunidad, han permitido que los efectos de la migración permeen de uno u otro modo en todos los hogares de la comunidad. Las especies que los migrantes nacionales traen en sus visitas se incorporan tanto en sus UD, como en aquellas de sus vecinos y familiares, y lo mismo ocurre con las ideas y conocimientos sobre el huerto de los migrantes nacionales e internacionales. Lo que demuestra que las redes sociales no sólo se dan hacia el interior de la comunidad, si no también hacia el exterior, al existir un constante intercambio de productos e información, fruto de la migración, pero sobre todo de la ubicación geográfica de la comunidad y la presencia de vías de comunicación adecuadas que permiten el rápido acceso a tres grandes ciudades del país como son el Distrito Federal, Toluca y Cuernavaca, desde tiempos remotos.
Bajo este complejo escenario, las familias atzincas han incorporado a su vida cotidiana una multiplicidad de actividades económicas y un manejo del espacio más allá de lo local, permitiendo que el huerto siga siendo un sistema multipropósito vigente. Sin embargo no hay que perder de vista que las circunstancias son cambiantes y que si bien en la actualidad la migración no ha ocasionado cambios tangibles entre huertos con migrantes y sin migrantes, hay circunstancias que podrían derivar en un escenario totalmente distinto. Hoy en día la migración de carácter temporal, está tendiendo a volverse internacional, definitiva y a incluir cada vez más al sector femenino por el endurecimiento de las políticas internacionales que dificultan el regreso de los migrantes, el fortalecimiento de las redes familiares y sociales en EU que facilita la llegada y establecimiento de los migrantes en dicho país, y la difícil situación económica de la comunidad que se ha recrudecido en los últimos años y que ha impulsado a muchos pobladores a tomar la decisión de migrar (Salazar-Barrientos, 2009). Por otra parte las políticas públicas regionales expresadas en el apoyo a programas productivos, como en el caso de los invernaderos de flor, podrían reorientar la estrategia diversificada de actividades hacia la ocupación mono-específica como una apuesta a una actividad momentáneamente exitosa, sobre todo porque en los últimos años la comunidad ha recibido cuantiosos apoyos gubernamentales por ser los "últimos indígenas tlahuicas", lo que de no manejarse adecuadamente, podría aumentar la dependencia económica de la comunidad y cambiar la relación huertos-migración.

Por ello para entender mejor esta relación proponemos analizar a mayor detalle las características del proceso migratorio, ya que la sola caracterización de las UD de acuerdo a la presencia o ausencia de migrantes no basta para comprender las consecuencias de este proceso. El número de migrantes por UD, así como el número de migraciones, el género, edad, destino, ocupación, temporalidad de la migración y rol del migrante dentro de la familia son variables a tomar en cuenta para un análisis más detallado de esta relación.

Finalmente consideramos que es indispensable continuar explorando junto a los pobladores estas relaciones complejas a partir de metodologías múltiples que nos permitan tener una perspectiva más amplia y multidimensional para así descubrir las distintas variables y sus matices que están interviniendo en los efectos que está teniendo la migración sobre la unidad doméstica y el huerto familiar, en particular en su estructura, composición y funciones.

\section{Agradecimientos}

Agradecemos el apoyo al Consejo Nacional para la Ciencia y la tecnología y al Programa de Posgrado en Ciencias Biológicas de la Universidad Nacional Autónoma de México para el desarrollo de este trabajo, así como a Heberto Ferrei- 
ra y Alberto Valencia, por su apoyo en el procesamiento de la información, a Eduardo García-Frapolli, Andrés Camou y Ernesto Vega por sus valiosas sugerencias, así como a los dos revisores anónimos por colaborar con sus observaciones a la mejora del manuscrito original. A Juan Martínez por el apoyo en la identificación de las especies de plantas. Y en especial a los pobladores de San Juan Atzingo y Lomas de Teocaltzingo, Estado de México.

\section{Literatura citada}

Agelet A., Bonet M.A. y Vallés J. 2000. Homegardens and their role as a main source of medicinal plants in mountain regions of Catalonia (Iberian Peninsula). Economic Botany 54:295-309.

Aguilar-Støen M., Moe S.R. y Camargo-Ricalde S.L. 2009. Home Gardens sustain crop diversity and improve farm resilience in Candelaria Loxicha, Oaxaca, México. Human Ecology 37:55-77.

Allison J.L. 1983. An ecological analysis of home Gardens (huertos familiares) in two mexican villages. Tesis de maestría University of California, Santa Cruz. 196 pp.

Álvarez-Fabela R.L. 2004. Tlahuicas. Serie Pueblos indígenas del México contemporáneo, Comisión Nacional para el Desarrollo de los Pueblos Indígenas. Disponible en línea: <www.cdi.gob. $\mathrm{mx} /$ index.php?option $=\mathrm{com} \_$docman $\&$ task $=$ doc_download $\& \mathrm{gi}$ $\mathrm{d}=52$ \&Itemid $=200020>$ (consultado abril 2008).

Appendini K. y Nuijten M. 2008. El papel de las instituciones en contextos locales: cuestiones metodológicas en investigación de campo. En: García-Barrios R., De la Tejera-Hernández B. y Appendini K. Coords. Instituciones y Desarrollo, Ensayos sobre la Complejidad del Campo Mexicano, pp. 251-280, Universidad Nacional Autónoma de México/Universidad Autónoma Chapingo/El Colegio de México, México, D.F.

Arzate-Salgado J. y Vizcarra-Bordi I. 2007. De la migración masculina transnacional: Violencia estructural y género en comunidades campesinas del Estado de México. Migración y Desarrollo 9:95-112.

Benjamin T.J., Montañez P.I., Jiménez J.J.M y Gillespie A.R. 2001. Carbon, water and nutrient flux in Maya homegardens in the Yucatán Peninsula of México. Agroforestry Systems 53:103-111

Blancas J., Casas A., Rangel-Landa S., Moreno-Calles A., Torres I., Pérez-Negrón E., Solís L., Delgado-Lemus A., Parra F., Arellanes Y., Caballero J., Cortés L., Lira R. y Dávila P. 2010. Plant management in the Tehuacán-Cuicatlán Valley, Mexico. Economic Botany 64:287-302.

Boege E. 2008. El Patrimonio Biocultural de los Pueblos Indígenas de México: Hacia la Conservación in situ de la Biodiversidad y Agrodiversidad en los Territorios Indígenas. Instituto Nacional de Antropología e Historia/ Comisión Nacional para el Desarrollo de los Pueblos Indígenas, México, D.F.

Basurto-Peña F.A. 1982. Huertos familiares en dos comunidades nahuas de la Sierra Norte de Puebla: Yancuictlalpan y Cuauhtapanaloyan. Tesis de licenciatura, Facultad de Ciencias Universidad Nacional Autónoma de México, México, D.F. 140 pp.

Caballero J. 1992. Maya homegardens: Past, present and future. Etnoecológica 1:35-54.

Cano-Ramírez M. 2003. Los huertos familiares de Tepango, Guerrero. Tesis de Licenciatura, Facultad de Ciencias Universidad Nacional Autónoma de México, México, D.F. 95 pp.
Casas A., Vázquez M.C., Viveros J.L. y Caballero J. 1996. Plant management among the Nahua and the Mixtec of the Balsas river basin: an ethnobotanical approach to the study of plant domestication. Human Ecology 24:455-478.

Casas A., Otero-Arnaiz A., Pérez-Negrón E. y Valiente-Banuet A. 2007. In situ management and domestication of plants in Mesoamerica. Annals of Botany 100:1101-1115.

Chi-Quej J.A. 2009. Caracterización y manejo de los huertos caseros familiares en tres grupos étnicos (Mayas peninsulares, Choles y Mestizos) del Estado de Campeche, México. Tesis de maestría, Escuela de Posgrado, Centro Agronómico Tropical de Investigación y Enseñanza, Turrialba, Costa Rica. 99 pp.

De Clerck F.A.J. y Negreros-Castillo, P. 2000. Plant species of traditional mayan homegardens of Mexico as analogs for multistrata agroforests. Agroforestry Systems 48:303-317.

De la Tejera-Hernández B. y García-Barrios R. 2008, Agricultura y estrategias de formación de ingreso campesinas en comunidades indígenas forestales oaxaqueñas. En: García-Barrios R., De la Tejera-Hernández B. y Appendini K. Coords. Instituciones y Desarrollo, Ensayos sobre la Complejidad del Campo Mexicano, pp. 65-103, Universidad Nacional Autónoma de México/Universidad Autónoma Chapingo/El Colegio de México, México, D.F.

Del Ángel-Pérez A.L. y Mendoza-B.M.A. 2004. Totonac homegardens and natural resources in Veracruz, Mexico. Agriculture and Human Values 21:329-346.

Ellis F. y Allison E. 2004. Livelihood diversification and natural resource access. LSP working paper 9, Food and Agriculture Organization of the United Nations, Norwich. Disponible en línea: <www.fao.org/es/esw/lsp/cd/img/docs/LSPWP9.pdf> (consultado enero 2011).

Engels J. 2002. Home gardens-a genetic resources perspective. En: Watson J.W. y Eyzaguirre P.B. 2002. Proceedings of the Second International Home Gardens Workshop: Contribution of home gardens to in situ conservation of plant genetic resources in farming systems, International Plant Genetic Resources Institute, Rome.

García de Miguel J. 2000. Etnobotánica maya: origen y evolución de los huertos familiares de la Península de Yucatán, México. Tesis doctoral, Universidad de Córdoba, Córdoba. 242 pp.

González-Ortiz F. 2005. Estudio Sociodemográfico de los Pueblos y Comunidades Indígenas del Estado de México, Consejo Estatal para el Desarrollo Integral de los Pueblos Indígenas del Estado de México/ El Colegio Mexiquense A.C., México.

Gotelli N.J. y Colwell R.K. 2001. Quantifying biodiversity: procedures and pitfalls in the measurement and comparison of species richness. Ecology Letters 4:379-391.

Guerrero-Peñuelas A.G. 2007. El impacto de la migración en el manejo de solares campesinos, caso de estudio La Purísima Concepción Mayorazgo, San Felipe del Progreso, Estado de México. Investigaciones Geográficas 63:105-124.

Hernández-Suárez C. y Vázquez-García V. 2007. La problemática socioambiental de la cuenca del río Texcoco. Una revisión de literatura. Agricultura, Sociedad y Desarrollo 4:39-52.

Howard P.L. 2006. Gender and social dynamics in swidden and homegardens in Latin America. En: Kumar B.M. y Nair P.K.R. Eds. Tropical Homergardens. A Time-Tested Example of Sustainable Agroforestry, pp. 159-182, Springer, Dordrecht.

IDRC [International Development Research Centre]. 2007. Annual Report 2006-2007. Disponible en línea: 〈http://www.idrc. 
ca/en/ev-100690-201-1-DO_TOPIC.html> (consultado octubre 2009).

INEGI [Instituto Nacional De Estadística y Geografía]. 2000. XII Censo General de Población y Vivienda. Disponible en línea: 〈http://www.inegi.org.mx/sistemas/olap/Proyectos/bd/censos/ cpv2000/PTL.asp?s=est\&c=10261\&proy=cpv00_pt > (consultado marzo 2007)

INEGI. 2010. Censo de Población y vivienda: datos preliminares. Disponible en línea: 〈http://www.inegi.org.mx/sistemas/consulta_resultados/iter2010.aspx?c=27329\&s=est > (consultado mayo 2011).

Kimber C.T. 2004. Gardens and dwelling: people in vernacular gardens. Geographical Review 94:263-283.

Korsbaek L. y Álvarez-Fabela R.L. 2002. Lengua y etnicidad: dos casos en el Estado de México. Convergencia 29:181-216.

Miller R.P., Penn J.W. y van Leeuwen J. 2006. Amazonian homegardens: their ethnohistory and potential contribution to agroforestry development. En: Kumar B.M. y Nair P.K.R. Eds. Tropical Homergardens. A Time-Tested Example of Sustainable Agroforestry, pp. 43-60, Springer, Dordrecht.

Moreno-Calles A., Casas A., Blancas J., Torres I., Masera O., Caballero J., García-Barrios L., Pérez-Negrón E. y Rangel-Landa S. 2010. Agroforestry systems and biodiversity conservation in arid zones: the case of the Tehuacan Valley, Central México. Agroforestry Systems 80:315-331.

Magurran A. 2004. Measuring Biological Diversity. Blackwell Publishing, Malden.

Manzanares-Medina G.I., Flores-Martínez A. y Hunn E.S. 2009. Los huertos familiares zapotecos de San Miguel Talea de Castro, Sierra Norte de Oaxaca, México. Etnobiología 7:9-29.

Nair P.K.R. 2006. Whither homegardens?. En: Kumar B.M. y Nair P.K.R. Eds. Tropical Homergardens. A Time-Tested Example of Sustainable Agroforestry, pp. 355-370, Springer, Dordrecht.

Orellana-Gallego R., Castiñeiras L., Fundadora Z., Shagarodsky T., Fuentes V., Barrios O., Cristóbal R., García M., Hernández F., García M., Giraudy C., Fernández L., Sánchez P., Moreno V. y Valiente A. 2006. Contribución de los huertos caseros rurales cubanos a la sostenibilidad ambiental.Cub@: Medio ambiente y desarrollo. Disponible en línea: http://www.medioambiente. cu/revistama/11_02.asp> (consultado junio 2010).

Pagaza-Calderón E.M. 2009. Efecto de la urbanización y el cambio cultural en la estructura florística de los huertos familiares y su papel en la conservación de especies silvestres, un estudio de caso en Tlacuilotepec, Puebla. Tesis de maestría, Posgrado en Ciencias Biológicas, Universidad Nacional Autónoma de México, México D.F. 83 pp.

Recibido: 12 de abril de 2011

Aceptado: 10 de noviembre de 2011
Parra F., Casas A., Peñaloza-Ramírez J.M., Cortés-Palomec A.C., Rocha-Ramírez V. y González-Rodríguez A. 2010. Evolution under domestication: ongoing artificial selection and divergence of wild and managed Stenocereus pruinosus (Cactaceae) populations in the Tehuacán Valley, Mexico. Annals of Botany 106:483-496.

Peyre A., Guidal A., Wiersum K.F. y Bongeres F. 2006. Dynamics of homegarden structure and function in Kerala, India. Agrofoerstry Systems 66:101-115.

Pulido M.T., Pagaza-Calderón E.M., Martínez-Ballesté A., Maldonado-Almanza B., Saynes A. y Pacheco R.M. 2008. Home Gardens as an alternative for sustainability: challenges and perspectives in Latin America. En: Albuquerque U.P y AlvesRamos M. Eds. Current Topics in Ethnobotany, pp.1-25, Research Signpost, Kerala.

Rebaï N. 2009. Migración, dinámicas territoriales y nueva ruralidad: un punto de vista desde la sierra ecuatoriana. Disponible en línea: <egal2009.easyplanners.info/area08/8155_Reba\%25ef_ Nasser.doc $>$ (consultado junio 2010).

Ruenes-Morales M.R. 1993. Estudio de los huertos familiares en los ejidos El Ahuacate y Adolfo López Mateos, de la Sierra de San Juan, Nayarit. Tesis de maestría, Facultad de Ciencias, Universidad Nacional Autónoma de México, México, D.F. 156 pp.

Salazar-Barrientos L.L. 2009. Migración y economía campesina: miradas para reflexionar en el caso de San Juan Atzingo, Mex. Tesis de maestría, Centro Regional Universitario Centro Occidente, Universidad Autónoma Chapingo, Morelia, Michoacán. $145 \mathrm{pp}$.

Vargas S.B. 2009. Ruralidades emergentes y dinámicas territoriales: nuevas percepciones y medios de vida. Eleuthera 3:194-205.

Vogl C.R., Vogl-Lukasser B.N. y Caballero J. 2002. Homegardens of maya migrants in the district of Palenque, Chiapas, Mexico. Implications for sustainable rural development. En: Stepp J.R, Wyndham F.S y Zarger R.K. Eds. Ethnobiology and Biocultural Diversity, pp. 631-647, University of Georgia Press, Georgia.

Wiersum K.F. 2006. Diversity and change in homegarden cultivation in Indonesia. En: Kumar B.M. y Nair P.K.R. Eds. Tropical Homergardens. A Time-Tested Example of Sustainable Agroforestry, pp. 13-24, Springer, Dordrecht.

Zarate-Vargas G. 2007. Estrategias campesinas de producción y comercialización de nopal y chícharo en la comunidad de San Juan Atzingo, estado de México: hacia una propuesta agroecológica y de desarrollo local. Tesis de maestría. Centro Regional Universitario Centro Occidente, Universidad Autónoma Chapingo, Morelia, Michoacán. 199 pp. 
Apéndice. Listado de plantas perennes de los huertos de San Juan Atzingo

\begin{tabular}{|c|c|c|}
\hline Familia & Nombre científico & Nombre común \\
\hline Acanthaceae & $\begin{array}{l}\text { Hypoestes sanguinolenta Hook. } \\
\text { Jacobinia spicigera L.H.Bailey } \\
\text { Pachystachys lutea Ness. } \\
\text { Thunbergia alata Bojer ex Sims }\end{array}$ & $\begin{array}{l}\text { *Sp. } 2 \\
\text { Muicle } \\
\text { *Sp. } 1 \\
\text { Ojo de cabra/O. pájaro/ O. canario }\end{array}$ \\
\hline Asparagaceae & $\begin{array}{l}\text { Agave salmiana Otto. } \\
\text { Agave tequilana F.A.C.Weber } \\
\text { Asparagus plumosus Baker } \\
\text { Asparagus sprengeri Regel } \\
\text { Cordyline rubra Heugel ex Kunth }\end{array}$ & $\begin{array}{l}\text { Maguey pulquero } \\
\text { Agave mezcalero } \\
\text { "Helecho" } \\
\text { Esparrago } \\
\text { Hoja sombra }\end{array}$ \\
\hline Altingiaceae & Liquidambar styraciflua L. & Liquidambar \\
\hline Aizoaceae & Aptenia cordifolia (L.f.) Schwantes & *Sp. 13 \\
\hline Aloaceae & $\begin{array}{l}\text { Aloe arborescens Mill. } \\
\text { Aloe aristata Haw. } \\
\text { Aloe juvenna Brandham \& S.Carter } \\
\text { Aloe saponaria Haw. } \\
\text { Aloe vera (L.) Burm. f. } \\
\text { Haworthia fasciata Haw. }\end{array}$ & $\begin{array}{l}\text { Sábila hembra } \\
\text { Cactus } \\
\text { Cactus } \\
\text { Sábila pinta } \\
\text { Sábila } \\
\text { *Sp. } 3\end{array}$ \\
\hline Amaranthaceae & Gomphrena serrata L. & Bolitas rosas \\
\hline Amaryllidaceae & $\begin{array}{l}\text { Agapanthus africanus Hoffmanns. } \\
\text { Hippeastrum sp. } \\
\text { Polianthes tuberosa L. }\end{array}$ & $\begin{array}{l}\text { Agapando } \\
\text { Azucena roja } \\
\text { Nardo }\end{array}$ \\
\hline Anacardiaceae & Schinus molle L. & Pirul \\
\hline Apiaceae & $\begin{array}{l}\text { Apium graveolens } \mathrm{L} . \\
\text { Foeniculum vulgare Miller. }\end{array}$ & $\begin{array}{l}\text { Apio } \\
\text { Hinojo }\end{array}$ \\
\hline Apocynaceae & $\begin{array}{l}\text { Catharanthus roseus (L.) G.Don } \\
\text { Mandevilla sanderi (Hemsl.) Woodson } \\
\text { Mandevilla x amabilis (hort. James Backhouse \& Son) Dress } \\
\text { Nerium oleander L. } \\
\text { Saintpaulia ionantha H.Wendl. }\end{array}$ & $\begin{array}{l}\text { Chino } \\
* \text { Sp. } 14 \\
\text { Adelfa } \\
\text { Violeta africana }\end{array}$ \\
\hline Araceae & $\begin{array}{l}\text { Anthurium andraeanum Linden } \\
\text { Colocasia esculenta (L.) Schott } \\
\text { Dieffenbachia bowmannii Carrière } \\
\text { Monstera obliqua Miq. } \\
\text { Philodendron scandens K.Koch \& Sello } \\
\text { Spathiphyllum wallisii Hort. } \\
\text { Zantedeschia aethiopica L. } \\
\text { Zantedeschia elliottiana Engl. }\end{array}$ & $\begin{array}{l}\text { Anturio } \\
\text { Hoja residencias/ H. elegante } \\
\text { Hoja de sombra } \\
\text { Calavera/ Esqueleto } \\
\text { Teléfono } \\
\text { Cuna de Moisés/ Cuna de Noé } \\
\text { Alcatraz } \\
\text { Alcatraz amarillo }\end{array}$ \\
\hline Araliaceae & $\begin{array}{l}\text { Hedera helix L. } \\
\text { Schefflera elegantissima (Veitch ex Masters) Lowry \& Frodin }\end{array}$ & $\begin{array}{l}\text { Hiedra } \\
\text { Sombra }\end{array}$ \\
\hline Araucariaceae & Araucaria heterophylla (Salisb) Franco & Araucaria \\
\hline Arecaceae & Syagrus romanzoffiana (Cham.) Glassman & Palma \\
\hline Asclepiadaceae & Ceropegia woodii Schltr. & Dos corazones en un hilo \\
\hline
\end{tabular}


Apéndice. Continuación

\begin{tabular}{|c|c|c|}
\hline Familia & Nombre científico & Nombre común \\
\hline Aspleniaceae & Asplenium monanthes $\mathrm{L}$. & Helecho 3 \\
\hline Asteraceae & $\begin{array}{l}\text { Argyranthemum frutescens Sch.Bip. } \\
\text { Artemisia absinthium L. } \\
\text { Artemisia ludoviciana Nutt. } \\
\text { Baccharis conferta Kunth } \\
\text { Barkleyanthus salicifolius (Kunth) H. Rob. \& Bretell } \\
\text { Bidens aurea (Aiton) Sherff } \\
\text { Bidens ballsii Sherff } \\
\text { Brickellia scoparia A.Gray } \\
\text { Calendula officinalis L. } \\
\text { Centaurea cineraria L. } \\
\text { Chrysantemum sp. } \\
\text { Chrysanthemum grandiflorum Hook. } \\
\text { Chrysanthemum maximum Ramond } \\
\text { Cirsium subcoriaceum Sch. Bip } \\
\text { Dahlia coccinea Cav. } \\
\text { Dahlia excelsa Benth. } \\
\text { Dahlia rudis P.D.Sørensen } \\
\text { Dimorphoteca ecklonis D.C } \\
\text { Euryops pectinatus Cass. } \\
\text { Gazania nivea Less. } \\
\text { Gerbera anandria Sch.Bip. } \\
\text { Heterotheca inuloides Cass. } \\
\text { Matricaria chamomilla L. } \\
\text { Melampodium sp. } \\
\text { Montanoa grandiflora Hemsl. } \\
\text { Montanoa tomentosa Cerv. } \\
\text { morfoespecie } 3 \\
\text { Pinaropappus roseus Less. } \\
\text { Piqueria trinervia Cav. } \\
\text { Stevia salicifolia Cav. } \\
\text { Tanacetum parthenium Sch.Bip. } \\
\text { Taraxacum officinale (L.) Weber } \\
\text { Bats }\end{array}$ & $\begin{array}{l}\text { "como crisantemo" } \\
\text { Ajenjo } \\
\text { Estafiate de casa y de monte } \\
\text { Escoba de popote/ Tepopote } \\
\text { Jara de monte } \\
\text { Acahual delgado } \\
\text { Acahual amarillo/ A. grueso } \\
\text { Prodigiosa } \\
\text { Coronilla } \\
\text { Espuma de mar } \\
\text { Polar } \\
\text { Crisantemo } \\
\text { Margaritón } \\
\text { Cardo } \\
\text { Dalia doble } \\
\text { Taxqueña/ Costeña } \\
\text { Dalia cimarrona } \\
\text { Margarita morada } \\
\text { Margarita canario } \\
\text { Novia del sol/ Agazania } \\
\text { Gerbera } \\
\text { Árnica } \\
\text { Manzanilla } \\
\text { Hierba de la clín } \\
\text { Girasol blanco } \\
\text { Zohuapacle } \\
\text { Ambar } \\
\text { Ixchpul } \\
\text { Hierba de San Nicolás } \\
\text { Hierba del aire } \\
\text { Santa maría } \\
\text { Diente de león/ Lechuguilla }\end{array}$ \\
\hline Balsaminaceae & Impatiens walleriana Hook. f. & Belén 2 \\
\hline Begoniaceae & $\begin{array}{l}\text { Begonia x tuberhybrida } \\
\text { Begonia coccinea Hook } \\
\text { Begonia gracilis Kunth } \\
\text { Impatiens balsamina L. }\end{array}$ & $\begin{array}{l}\text { Tuberosa } \\
\text { Ala de ángel } \\
\text { Begonia } \\
\text { Belén } 1\end{array}$ \\
\hline Bignonaceae & $\begin{array}{l}\text { Myosotis sylvatica Hoffm. } \\
\text { Podranea ricasoliana Sprague } \\
\text { Tecoma stans (L.) Kunth } \\
\text { Tournefortia densiflora Mart.et Galeotti }\end{array}$ & $\begin{array}{l}\text { No me olvides } \\
\text { Enredadera } 2 \\
* \text { Sp. } 8 \\
\text { Hierba rasposa }\end{array}$ \\
\hline Bromeliaceae & $\begin{array}{l}\text { Aechmea fasciata (Lindl.) } \\
\text { Ananas comosus (L.) Merr. } \\
\text { Tillandsia usneoides L. }\end{array}$ & $\begin{array}{l}\text { Planta elegante } \\
\text { Piña } \\
\text { Heno }\end{array}$ \\
\hline Buddlejaceae & $\begin{array}{l}\text { Buddleja perfoliata Kunth } \\
\text { Buddleja cordata Kunth } \\
\text { Buddleja sessiliflora Kunth }\end{array}$ & $\begin{array}{l}\text { Tepozán } \\
\text { Tepozán de San Juan } 2 \\
\text { Tepozán de San Juan } 1\end{array}$ \\
\hline Cactaceae & Aporocactus flagelliformis Lem. & * Sp. 9 \\
\hline
\end{tabular}


Apéndice. Continuación

\begin{tabular}{|c|c|c|}
\hline Familia & Nombre científico & Nombre común \\
\hline & $\begin{array}{l}\text { Austrocylindropuntia subulata (Engelm.) Backeb. } \\
\text { Cereus forbesii Hort.Berol. ex Foerst. } \\
\text { Chamaecereus silvestrii Britton \& Rose } \\
\text { Echinopsis eyriesii Pfeiff. \& Otto } \\
\text { Heliocereus speciosus Britton \& Rose } \\
\text { Hylocereus undatus Britton \& Rose } \\
\text { Mammillaria sp. } \\
\text { Mammillaria spinosissima Salm-Dyck } \\
\text { Marginatocereus marginatus (DC.) Backeb. } \\
\text { Opuntia ficus-indica (L) Mill. } \\
\text { Opuntia microdasys (Lehm.) Pfeiff } \\
\text { Opuntia sp. } \\
\text { Rhipsalidopsis gaertneri (K.Schum.) Linding. }\end{array}$ & $\begin{array}{l}\text { Cactus } 2 \\
\text { Cactus Felipa } 3 \\
\text { Cacahuate } \\
\text { Cactus juliana I } \\
\text { Pitahaya } 1 \\
\text { *Sp. } 4 \\
\text { Piña/ Bisnaga de monte } \\
\text { Piñita comprada } \\
\text { Órgano } \\
\text { Nopal verdura var. Milpa Alta } \\
\text { Cactus Genoveva } 1 \\
\text { Nopalillo } \\
\text { Pitahayita }\end{array}$ \\
\hline Campanulaceae & Lobelia laxiflora Kunth & Aretillo cimarrón/ La suegra \\
\hline Cannaceae & Canna indica L. & Platanillo \\
\hline Caprifoliaceae & Lonicera japonica Thunb. & Madre selva \\
\hline Caryophyllaceae & $\begin{array}{l}\text { Dianthus caryophyllus L. } \\
\text { Dianthus deltoides L. } \\
\text { Lychnis coronaria (L.) Desr. }\end{array}$ & $\begin{array}{l}\text { Clavel } \\
\text { Clavellina } \\
\text { Violeta }\end{array}$ \\
\hline Celastraceae & Euonymus japonicus Thunb. & Ebónimo \\
\hline Clusiaceae & Hypericum calycinum L. & Mariposa \\
\hline Commelinaceae & $\begin{array}{l}\text { Commelina diffusa Burm.f. } \\
\text { Gibasis geniculata (Jacq.) Rohweder } \\
\text { Tradescantia pallida (Rose) D.R.Hunt }\end{array}$ & $\begin{array}{l}* \text { Sp. } 5 \\
* \text { Sp } 5 \\
* \text { Sp } 15\end{array}$ \\
\hline Convulvulaceae & Ipomea sp. & Estafiate de monte \\
\hline Crassulaceae & $\begin{array}{l}\text { Aeonium arboreum Webb \& Berthel. } \\
\text { Echeveria secunda Booth ex Lindl. } \\
\text { Echeveria shaviana E.Walther } \\
\text { Kalanchoe blossfeldiana Poelln. } \\
\text { Kalanchoe serrata Mannoni \& Boiteau } \\
\text { Kalanchoe tomentosa Baker } \\
\text { Sedum burrito R.Moran } \\
\text { Sedum dasyphyllum L. } \\
\text { Sedum dendroideum DC. } \\
\text { Sedum greggii Hemsl. } \\
\text { Sedum morganianum E. Walther } \\
\text { Sedum oxypetalum Kunth. } \\
\text { Sedum pachucense Praeger } \\
\text { Sedum pachyphyllum Rose } \\
\text { Sedum quevae Raym. Hamet }\end{array}$ & $\begin{array}{l}\text { Conchita grande } \\
\text { Magueyito de las peñas } \\
\text { Oreja de burro/ Hoja de } \\
\text { lechuga clara } \\
\text { Coral/ Anchoa } \\
\text { Cola de lagartija } \\
\text { *Sp. } 16 \\
\text { Cola de zorro } \\
\text { Piojito } \\
\text { Del monte } \\
\text { Conchita chica de monte } \\
\text { Cola de borrego } \\
\text { Oreja de ratón } \\
\text { Rocío } \\
\text { Deditos de dios/ Manita/ } \\
\text { Siempre vive } \\
\text { Chisme/ Estrellita }\end{array}$ \\
\hline Cruciferae & Matthiola incana (L.) W.T.Aiton & Alelía \\
\hline Cucurbitaceae & Sechium edule Sw. & Chayote \\
\hline
\end{tabular}


Apéndice. Continuación

\begin{tabular}{|c|c|c|}
\hline Familia & Nombre científico & Nombre común \\
\hline Cupresaceae & $\begin{array}{l}\text { Chamaecyparis pisifera (Siebold \& Zucc.) Endl. } \\
\text { Cupressus macrocarpa A.Cunn. } \\
\text { Cupressus sempervirens L. } \\
\text { Juniperus deppeana Steud. }\end{array}$ & $\begin{array}{l}\text { Pino chimancipare } \\
\text { Cedrito limón } \\
\text { Cipres italiano } \\
\text { Cedro }\end{array}$ \\
\hline Davalliaceae & $\begin{array}{l}\text { Nephrolepis exaltata (L.) Schott } \\
\text { Nephrolepis sp. }\end{array}$ & $\begin{array}{l}\text { Helecho } 4 \\
\text { Helecho } 5\end{array}$ \\
\hline Dracaenaceae & $\begin{array}{l}\text { Dasylirion sp. } \\
\text { Dracaena deremensis Engl. } \\
\text { Dracaena fragrans (L.) Ker Gawl. } \\
\text { Dryopteris wallichiana (Spreng.) Hyl. } \\
\text { Plecosorus speciosissimus (A.Braun) T.Moore }\end{array}$ & $\begin{array}{l}\text { Maguey cucharilla/ M. de la } \\
\text { virgen/M. para portadas } \\
\text { *Sp. } 6 \\
\text { Hoja rayitas } \\
\text { Palmita } \\
\text { Helecho } 2\end{array}$ \\
\hline Ericaceae & $\begin{array}{l}\text { Arctostaphylos pungens Kunth } \\
\text { Azalea japonica A.Gray }\end{array}$ & $\begin{array}{l}\text { Pingüica } \\
\text { Azalea }\end{array}$ \\
\hline Euphorbiaceae & $\begin{array}{l}\text { Acalypha hispida Willd. } \\
\text { Euphorbia milii Des Moul. } \\
\text { Euphorbia pulcherrima Willd. ex Klotzsch } \\
\text { Euphorbia trigona Mill. } \\
\text { Ricinus comunis L. }\end{array}$ & $\begin{array}{l}\text { Gusanito } \\
\text { Corona de Cristo } \\
\text { Nochebuena } \\
\text { Cactus } \\
\text { *Sp. } 18\end{array}$ \\
\hline Fabaceae & $\begin{array}{l}\text { Erythrina americana Mill. } \\
\text { Senna multiglandulosa (Jacquin) H.S.Irwin \& Barneby } \\
\text { Trifolium mexicanum Hemsl. }\end{array}$ & $\begin{array}{l}\text { Colorín/ Zopacle } \\
\text { Retama } \\
\text { Xocoyol de conejo/ (trébol normal) }\end{array}$ \\
\hline Fagaceae & $\begin{array}{l}\text { Quercus laurina Humb. \& Bonpl. } \\
\text { Quercus rugosa Née }\end{array}$ & $\begin{array}{l}\text { Encino delgado } \\
\text { Encino grueso }\end{array}$ \\
\hline Gentianaceae & Gentiana spathacea Kunth & Mira cielo \\
\hline Geraniaceae & $\begin{array}{l}\text { Geranium seemannii Peyr. } \\
\text { Pelargonium peltatum (L.) L'Hér. } \\
\text { Pelargonium x domesticum L.H. Bailey }\end{array}$ & $\begin{array}{l}\text { Pata de león/ Mano de león/ Pata } \\
\text { de gallo } \\
\text { Geranio } \\
\text { Malvón }\end{array}$ \\
\hline Hemerocallidaceae & Hemerocallis flava L. & Lirio amarillo \\
\hline Hydrangeaceae & $\begin{array}{l}\text { Hydrangea hortensia Siebold } \\
\text { Philadelphus mexicanus Schltdl. }\end{array}$ & $\begin{array}{l}\text { Hortensia } \\
\text { Jazmín doble }\end{array}$ \\
\hline Iridaceae & $\begin{array}{l}\text { Crocosmia Planch } \\
\text { Gladiolus sp. } \\
\text { Iris germanica L. }\end{array}$ & $\begin{array}{l}\text { Agapando naranja/ Leonora } \\
\text { Gladiola/ Palmira } \\
\text { Lirio } 1\end{array}$ \\
\hline Juglandaceae & Juglans regia $\mathrm{L}$. & Nogal \\
\hline Labiatae & $\begin{array}{l}\text { Agastache mexicana (Kunth) Lint \& Epling } \\
\text { Lepechinia caulescens (Ortega) Epling } \\
\text { Melissa officinalis L. } \\
\text { Origanum majorana L. } \\
\text { Salvia mexicana L. } \\
\text { Thymus vulgaris L. }\end{array}$ & $\begin{array}{l}\text { Toronjil de casa } \\
\text { Bretónica/ Sonajilla } \\
\text { Toronjil } \\
\text { Mejorana de monte } \\
\text { Chía corriente } \\
\text { Tomillo }\end{array}$ \\
\hline
\end{tabular}


Apéndice. Continuación

\begin{tabular}{|c|c|c|}
\hline Familia & Nombre científico & Nombre común \\
\hline Lamiaceae & $\begin{array}{l}\text { Coleus blumei Benth. } \\
\text { Marrubium vulgare L. } \\
\text { Mentha viridis (L.) L. } \\
\text { Origanum vulgare L. } \\
\text { Plectranthus australis R.Br. } \\
\text { Plectranthus cylindraceus Hochst. } \\
\text { Rosmarinus officinalis L. } \\
\text { Salvia gesneraflora Lindl. } \\
\text { Salvia iodantha Fernald } \\
\text { Salvia lavanduloides Kunth. } \\
\text { Salvia microphylla Kunth }\end{array}$ & $\begin{array}{l}\text { *Sp. } 16 \\
\text { Marrubio } \\
\text { Hierbabuena } \\
\text { Orégano } \\
\text { Moneda } \\
\text { Vaporub } \\
\text { Romero } \\
\text { Mirto rojo grande/ M. cimarrón } \\
\text { (silvestre) } \\
\text { Mirto rojo chico } \\
\text { Chía } 2 \\
\text { Mirto rosa de casa }\end{array}$ \\
\hline Lauraceae & $\begin{array}{l}\text { Laurus nobilis L. } \\
\text { Persea americana Mill. }\end{array}$ & $\begin{array}{l}\text { Laurel } \\
\text { Aguacate }\end{array}$ \\
\hline Leguminosae & $\begin{array}{l}\text { Acacia longifolia (Andrews) Willd. } \\
\text { Inga spuria Humb. \& Bonpl. ex Willd. }\end{array}$ & $\begin{array}{l}* \text { Sp. } 5 \\
\text { Cajinicuil }\end{array}$ \\
\hline Liliaceae & $\begin{array}{l}\text { Chlorophytum comosum (Thunb.) Jacques } \\
\text { Hyacinthus orientalis L. } \\
\text { Kniphofia uvaria (L.) Oken } \\
\text { Lilium candidum L. } \\
\text { Lilium sp. } \\
\text { Lilium speciosum Thunb. } \\
\text { Tigridia pavonia (L.f.) DC. }\end{array}$ & $\begin{array}{l}\text { Mala madre } \\
\text { Jacinto } \\
\text { Mazorquita naranja } \\
\text { Azucena rosa } \\
\text { Lili } \\
\text { Acapulco } \\
\text { Lirio } 2\end{array}$ \\
\hline Lobeliaceae & Lobelia erinus L. & Mosquito \\
\hline Lythraceae & $\begin{array}{l}\text { Cuphea hyssopifolia Kunth } \\
\text { Heimia salicifolia Link \& Otto } \\
\text { Punica granatum L. }\end{array}$ & $\begin{array}{l}* \text { Sp. } 8 \\
\text { Chinicuil } \\
\text { Granada roja }\end{array}$ \\
\hline Malvaceae & $\begin{array}{l}\text { Abutilon hybridum Hort. } \\
\text { Hibiscus rosa-sinensis L. } \\
\text { Malva aff. sylvestris } \\
\text { Sphaeralcea angustifolia G.Don }\end{array}$ & $\begin{array}{l}\text { Linda tarde } \\
\text { Tulipán } \\
\text { Malva de casa } \\
\text { Hierba del negro }\end{array}$ \\
\hline Melastromataceae & Tibouchina urvilleana (DC.) Cogn. & Martha \\
\hline Moraceae & $\begin{array}{l}\text { Ficus carica L. } \\
\text { Ficus microcarpa Hort.Berol. ex Walp. }\end{array}$ & $\begin{array}{l}\text { Higo } \\
* \text { Sp. } 9\end{array}$ \\
\hline Musaceae & Mussa paradisiaca L. & Plátano \\
\hline Myrtaceae & $\begin{array}{l}\text { Bougainvillea spectabilis Wild. } \\
\text { Callistemon citrinus Stapf } \\
\text { Eucalyptus cinerea F.Muell. ex Benth. } \\
\text { Eucalyptus globulus Labill } \\
\text { Psidium guajava L. }\end{array}$ & $\begin{array}{l}\text { Bugambilia } \\
\text { *Sp. } 10 \\
\text { Dólar } \\
\text { Eucalipto/ Alcanfor } \\
\text { Guayaba }\end{array}$ \\
\hline Nictaginaceae & Mirabilis jalapa L. & Maravilla del campo \\
\hline Onagraceae & $\begin{array}{l}\text { Fuchsia magellanica Lam. } \\
\text { Fuchsia microphylla Kunth. } \\
\text { Lopezia racemosa Cav. }\end{array}$ & $\begin{array}{l}\text { Aretillo } \\
\text { Aretillo miniatura } \\
\text { Lujo silvestre }\end{array}$ \\
\hline
\end{tabular}


Apéndice. Continuación

\begin{tabular}{|c|c|c|}
\hline Familia & Nombre científico & Nombre común \\
\hline Orchidaceae & Laelia sp. & Orquídea rosa \\
\hline Oxalidaceae & $\begin{array}{l}\text { Oxalis alpina Rose ex R.Knuth } \\
\text { Oxalis latifolia Kunth } \\
\text { Oxalis pes-caprae L. }\end{array}$ & $\begin{array}{l}\text { Xocoyol de casa } \\
\text { Xocoyol blanco } \\
\text { Xocoyol de milpa }\end{array}$ \\
\hline Passifloraceae & $\begin{array}{l}\text { Passiflora ligularis L. } \\
\text { Passiflora mollissima L.H.Bailey }\end{array}$ & $\begin{array}{l}\text { Granada moco } \\
\text { Granada agria }\end{array}$ \\
\hline Pinaceae & $\begin{array}{l}\text { Abies religiosa (Kunth) Schltdl. \& Cham. } \\
\text { Pinnus teocote L. }\end{array}$ & $\begin{array}{l}\text { Oyamel } \\
\text { Ocote de monte }\end{array}$ \\
\hline Piperaceae & $\begin{array}{l}\text { Peperomia caperata Yunck. } \\
\text { Pipper auritum Kunth. } \\
\text { Plantago australis Lam. }\end{array}$ & $\begin{array}{l}\text { Cascara de nuez/hoja de nuez } \\
\text { Hierbasanta } \\
\text { Lantén }\end{array}$ \\
\hline Poaceae & $\begin{array}{l}\text { Cymbopogon citratus Stapf } \\
\text { Phyllostachys aurea Carrière ex Rivière \& C.Rivière }\end{array}$ & $\begin{array}{l}\text { Zacate limón } \\
\text { Bambú/junco }\end{array}$ \\
\hline Polygonaceae & $\begin{array}{l}\text { Rumex crispus L. } \\
\text { Rumex mexicanus L. }\end{array}$ & $\begin{array}{l}\text { Lengua de vaca fina } \\
\text { Lengua de vaca corriente }\end{array}$ \\
\hline Portulacaceae & Portulacaria afra Jacq. & $\begin{array}{l}\text { Árbol abundancia/ A. suerte/ } \\
\text { A. fortuna }\end{array}$ \\
\hline Primulaceae & Primula vulgaris Huds. & *Sp. 7 \\
\hline Pteridaceae & Cheilanthes bonariensis (Willd.) Proctor & Helecho 1 \\
\hline Ranunculaceae & $\begin{array}{l}\text { Aquilegia vulgaris L. } \\
\text { Clematis dioica L. }\end{array}$ & $\begin{array}{l}\text { Aquilegia } \\
\text { Jazmín sencillo }\end{array}$ \\
\hline Rosaceae & $\begin{array}{l}\text { Crataegus pubescens Steud. } \\
\text { Duchesnea indica (Andrews) Focke } \\
\text { Eriobotrya japonica (Thunb.) Lindl. } \\
\text { Esenbeckia sp. } \\
\text { Fraxinus excelsior L. } \\
\text { Malus domestica Borkh. } \\
\text { Prunus armeniaca L. } \\
\text { Prunus cerasifera Ehrh. } \\
\text { Prunus domestica L. } \\
\text { Prunus persica (L.) Batsch } \\
\text { Prunus salicina Lindl. } \\
\text { Prunus serotina Ehrh. } \\
\text { Pyrus communis L. } \\
\text { Pyrus malus L. } \\
\text { Rosa centifolia L. } \\
\text { Rosa sp. } \\
\text { Rubus fruticosus L. } \\
\text { Rubus idaeus L. }\end{array}$ & $\begin{array}{l}\text { Tejocote } \\
\text { Frecita de monte } \\
\text { Níspero } \\
\text { Clavo } \\
\text { Fresno } \\
\text { Manzana } \\
\text { Chabacano } \\
\text { Ciruelo pasa } \\
\text { Ciruelo rojo } \\
\text { Durazno } \\
\text { Ciruelo amarillo } \\
\text { Capulín } \\
\text { Pera } \\
\text { Perón } \\
\text { Rosa castilla } \\
\text { Rosa } \\
\text { Zarza cultivada y zarza de lindero } \\
\text { Frambuesa }\end{array}$ \\
\hline Rutaceae & $\begin{array}{l}\text { Casimiroa edulis La Llave } \\
\text { Citrus sinensis Pers. } \\
\text { Citrus limetta Risso } \\
\text { Citrus limon L. }\end{array}$ & $\begin{array}{l}\text { Zapote blanco } \\
\text { Naranja } \\
\text { Lima } \\
\text { Limón }\end{array}$ \\
\hline
\end{tabular}


Apéndice. Continuación

\begin{tabular}{|c|c|c|}
\hline Familia & Nombre científico & Nombre común \\
\hline & $\begin{array}{l}\text { Citrus nobilis Lour. } \\
\text { Ruta graveolens L. }\end{array}$ & $\begin{array}{l}\text { Mandarina agria } \\
\text { Ruda }\end{array}$ \\
\hline Plantaginaceae & $\begin{array}{l}\text { Antirrhinum majus L. } \\
\text { Cymbalaria muralis G.Gaertn., B.Mey. \& Schreb. } \\
\text { Datura sanguinea Ruiz \& Pav. } \\
\text { Digitalis purpurea L. } \\
\text { Lophospermum scandens Sessé \& Moc. Ex D. Don } \\
\text { Maurandya scandens Pers. } \\
\text { Penstemon campanulatus Willd } \\
\text { Penstemon roseus G.Don }\end{array}$ & $\begin{array}{l}\text { Perrito de casa } \\
\text { Greñas de mi suegra } \\
\text { Florifundio } \\
\text { Perrito de monte } \\
\text { Flor de lujo del cerro } \\
\text { Flor Sofía } \\
\text { Jarrita del monte } \\
\text { Jarrita chiquita }\end{array}$ \\
\hline Sapindaceae & Serjania triquetra Radkl. & Tres costillas \\
\hline Solanaceae & $\begin{array}{l}\text { Bouchetia sp. } \\
\text { Cestrum anagyris Dunal } \\
\text { Cestrum nocturnum L. } \\
\text { Jaltomata procumbens L. } \\
\text { Lycopersicum esculentum Mill. } \\
\text { Petunia } \times \text { hybrida E.Vilm. }\end{array}$ & $\begin{array}{l}\text { *Sp. } 17 \\
\text { Hierba negra } \\
\text { Huele de noche } \\
\text { Jaltomate morado } \\
\text { Jitomate } \\
\text { Petunia }\end{array}$ \\
\hline Strelitziaceae & Strelitzia reginae Banks & Ave del paraíso \\
\hline Taxodiaceae & Taxodium mucronatum Ten. & Ahuehuete \\
\hline Theaceae & $\begin{array}{l}\text { Camellia japonica L. } \\
\text { Camellia sinensis (L.) Kuntze }\end{array}$ & $\begin{array}{l}\text { *Sp. } 11 \\
\text { Té negro }\end{array}$ \\
\hline Tropaeolaceae & Tropaeolum majus L. & *Sp. 12 \\
\hline Urticaceae & $\begin{array}{l}\text { Soleirolia soleirolii (Req.) Dandy } \\
\text { Urtica mexicana Blume }\end{array}$ & $\begin{array}{l}\text { Alfombra } \\
\text { Chichicaxtle }\end{array}$ \\
\hline Verbenaceae & $\begin{array}{l}\text { Aloysia citrodora Paláu } \\
\text { Lantana camara L. } \\
\text { Priva mexicana Sieber ex Steud. }\end{array}$ & $\begin{array}{l}\text { Cedrón } \\
\text { Mil amores/ La nuera y la suegra } \\
\text { Verbena }\end{array}$ \\
\hline Violaceae & Viola tricolor L. & Pensamiento \\
\hline Vitaceae & Vitis vinifera L. & Uva \\
\hline
\end{tabular}

